\title{
Activity of Human Dihydrolipoamide Dehydrogenase Is Largely Reduced by Mutation at Isoleucine-51 to Alanine
}

\author{
Hakjung Kim* \\ Department of Chemistry, College of Natural Science, Daegu University, Kyoungsan 712-714, Korea
}

Received 9 November 2005, Accepted 18 January 2006

\begin{abstract}
Dihydrolipoamide dehydrogenase (E3) belongs to the pyridine nucleotide-disulfide oxidoreductase family including glutathione reductase and thioredoxin reductase. It catalyzes the reoxidation of dihydrolipoyl moiety of the acyltransferase components of three $\alpha$-keto acid dehydrogenase complexes and of the hydrogen-carrier protein of the glycine cleavage system. Isoleucine-51 of human E3, located near the active disulfide center Cys residues, is highly conserved in most E3s from several sources. To examine the importance of this highly conserved Ile-51 in human E3 function, it was substituted with Ala using site-directed mutagenesis. The mutant was expressed in Escherichia coli and highly purified using an affinity column. Its E3 activity was decreased about $\mathbf{1 0 0}$-fold, indicating that the conservation of the Ile-51 residue in human $\mathrm{E3}$ was very important to the efficient catalytic function of the enzyme. Its altered spectroscopic properties implied that conformational changes could occur in the mutant.
\end{abstract}

Keywords: Dihydrolipoamide dehydrogenase, $\alpha$-keto acid dehydrogenase complex, Pyridine nucleotide-disulfide oxidoreductase family, Site-directed mutagenesis, Structural homology

\section{Introduction}

Dihydrolipoamide dehydrogenase (E3) (dihydrolipoamide:NAD ${ }^{+}$ oxidoreductase; EC 1.8.1.4) along with glutathione reductase (GR), thioredoxin reductase, and trypanothione reductase belong to the pyridine nucleotide-disulfide oxidoreductase family (William, 1976). All of them have homodimeric structures containing an active disulfide center and a FAD in each subunit. Through the FAD and active disulfide center,

*To whom correspondence should be addressed.

Tel: 82-53-850-6447; Fax: 82-53-850-6449

E-mail: hjkim@daegu.ac.kr they catalyze electron transfers between pyridine nucleotides $\left(\mathrm{NAD}^{+}\right.$or NADPH) and their specific substrates.

Each subunit of human E3 consists of 474 amino acids with a molecular mass of 50,216 daltons calculated from the primary amino acid sequence (Pons et al., 1988). It is a common component in three $\alpha$-keto acid dehydrogenase complexes (pyruvate, $\alpha$-ketoglutarate and branched-chain $\alpha$ keto acid dehydrogenase complexes) (Reed, 1974) and the glycine cleavage system (Walker and Oliver, 1986). It catalyzes the reoxidation of the dihydrolipoyl prosthetic group attached to the lysyl residue(s) of the acyltransferase components of the three $\alpha$-keto acid dehydrogenase complexes and to the hydrogen-carrier protein of the glycine cleavage system.

The structure of the human E3 active site has been proposed (Jentoft et al., 1992) on the basis of the threedimensional structures of human GR (Thieme et al., 1981) and of Azotobacter vinelandii E3 (Schierbeek et al., 1989). The cDNA sequence for human E3 has been cloned (Pons et al., 1988) and expressed in E. coli (Kim et al., 1991). The recombinant human E3 possessed similar properties to those of purified mammalian E3s. The putative essential amino acid residues of human E3 have been modified using site-directed mutagenesis and the mutants have been characterized (Kim and Patel, 1992; Leu et al., 1995; Kim, 1999b; Kim, 2002; Kim, 2005).

The active disulfide center plays a critical role in the catalysis of pyridine nucleotide-disulfide oxidoreductases having similar catalytic mechanisms. The active disulfide center of Azotobacter vinelandii $\mathrm{E} 3$ shows an unusual structure where the $\mathrm{C}^{\alpha}-\mathrm{C}^{\alpha}$ distance is relatively shorter than normal value (Mattevi et al., 1991). The geometric strain may be necessary for the breakage of the disulfide center during the catalysis.

The residues around the active disulfide centers of most pyridine nucleotide-disulfide oxidoreductases are highly conserved. The consensus sequence for the active disulfide center region of $34 \mathrm{E} 3 \mathrm{~s}$ having known primary structures from various sources was searched and the consensus sequence of GG(TV)CLN(VX)GCIP was suggested (Kim, 2001). The 10th Ile residue was highly conserved in most E3s from various 
sources. To examine the importance of this highly conserved Ile residue in E3 structure and function, the corresponding Ile51 residue in human E3 was substituted with Ala by sitedirected mutagenesis. E3 mutant was expressed in Escherichia coli and highly purified using an affinity column. The mutant possessed about 100-fold decreased E3 activity and altered spectroscopic properties.

\section{Materials and Methods}

Materials. The electrophoresis reagents, imidazole, iminodiacetic acid sepharose 6B, lipoamide and $\mathrm{NAD}^{+}$were from Sigma Chemical Co. Dihydrolipoamide was synthesized by reduction of lipoamide using sodium borohydride. Isopropyl- $\beta$-D-thiogalactopyranoside (IPTG) was from POSCOCHEM R\&D Center. E. coli XL1-Blue containing a human E3 expression vector pPROEX-1:E3 was a generous gift from Dr. Mulchand S. Patel of State University of New York at Buffalo. Pfu polymerase, other enzymes, primers and $\mathrm{dNTP}$ were from Bioneer.

\section{Site-directed mutagenesis and construction of the human E3} mutant expression vector pPROEX-1:E3 $(\mathbf{I}-51 \rightarrow$ A). Site-directed mutagenesis and the construction of the mutant expression vector were performed as described below. Two polymerase chain reactions (PCR) were carried out using the primer pairs A with $\mathrm{B}$ and $\mathrm{C}$ with D, respectively. Primer A (5'-TTACGATATCCCAACG ACCG-3') and Primer D (5'-GCCAAAACAAGCCAAGCTTGG$\left.3^{\prime}\right)$ are sense and anti-sense oligomers, respectively, which can be used to amplify the whole E3 gene sequence. Primer B (5'-TGC TTGAATGTTGGTTGTGCTCCTTCTAAGGCTTTATTG-3': the mismatched bases are underlined) is an anti-sense oligomer with point mutations to convert Ile-51 (ATT) to Ala (GCT). Primer C (5'-CAATAAAGCCTTAGAAGGACGACAACCAACATTCAAG CA-3': the mismatched bases are underlined) is the corresponding sense oligomer of the primer $\mathrm{B}$.

The reactions were performed using the human E3 expression vector pPPROEX-1:E3 as a template with $P f u$ polymerase in a programmable PCR machine (Kim, 2002). After a 5 min incubation at $95^{\circ} \mathrm{C}$, thirty three rounds of temperature cycling were performed as $95^{\circ} \mathrm{C}$ for $30 \mathrm{sec}, 43^{\circ} \mathrm{C}$ for $1 \mathrm{~min}, 72^{\circ} \mathrm{C}$ for $90 \mathrm{sec}$ and a final 5 min incubation at $72^{\circ} \mathrm{C}$ was followed. As expected, the PCR with primers $\mathrm{A}$ and $\mathrm{B}$ produced about a $220 \mathrm{bp}$ fragment $\mathrm{AB}$ while the PCR with primers $C$ and D generated about a 1200 bp fragment $\mathrm{CD}$. The fragments $\mathrm{AB}$ and $\mathrm{CD}$ were combined and used as a template for the subsequent PCR with primers A and D. After a 5 min incubation at $94^{\circ} \mathrm{C}$, five rounds of temperature cycling were performed as $94^{\circ} \mathrm{C}$ for $30 \mathrm{sec}$ and $72^{\circ} \mathrm{C}$ for $90 \mathrm{sec}$. Thirty rounds of temperature cycling were then followed at $94^{\circ} \mathrm{C}$ for $30 \mathrm{sec}, 58^{\circ} \mathrm{C}$ for $1 \mathrm{~min}, 72^{\circ} \mathrm{C}$ for $90 \mathrm{sec}$ and a final $5 \mathrm{~min}$ incubation at $72^{\circ} \mathrm{C}$ was carried out. This PCR generated a 1501 bp fragment AD which contained the E3 sequence with the mutation.

The fragment AD was digested with Mly113I and EcoRI and then the Mly113I/EcoRI fragment (909 bp) was isolated by agarose gel electrophoresis. A mutant expression vector, pPROEX-1:E3(S$79 \rightarrow \mathrm{C}$ ), was digested with Mly113I and EcoRI to remove the corresponding normal Mly113I/EcoRI fragment (Kim, 1999a). The vector lacking the Mly113I/EcoRI sequence was isolated by agarose gel electrophoresis and ligated with the previously isolated $M l y 113 \mathrm{I} / E c o$ RI fragment containing the mutation. The ligation resulted in the construction of the Ala-51 mutant expression vector, pPROEX-1:E3 $(\mathrm{I}-51 \rightarrow \mathrm{A})$. The mutation was confirmed by DNA sequencing.

Expression and purification of the human E3 mutant. Three $\mathrm{ml}$ of an overnight culture of $E$. coli XL1-Blue containing the human E3 mutant expression vector were used to inoculate $600 \mathrm{ml}$ of LB medium containing ampicillin $(100 \mu \mathrm{g} / \mathrm{ml})$. Cells were grown at $37^{\circ} \mathrm{C}$ to an absorbance of 0.7 at $595 \mathrm{~nm}$ and IPTG was added to a final concentration of $1 \mathrm{mM}$. The growing temperature was shifted to $30^{\circ} \mathrm{C}$ and cells were allowed to grow overnight. The overnight culture was harvested by centrifugation at $4000 \times g$ for $5 \mathrm{~min}$. Cell pellets were washed with $50 \mathrm{mM}$ potassium phosphate buffer $(\mathrm{pH}$ 8.0) containing $100 \mathrm{mM} \mathrm{NaCl}$ (Binding buffer) and then recollected by centrifugation at $4000 \times g$ for $5 \mathrm{~min}$. They were resuspended in $10 \mathrm{ml}$ of Binding buffer. They were lysed by a sonication treatment and centrifuged at $10,000 \times g$ for $20 \mathrm{~min}$.

The supernatant was loaded on a nickel iminodiacetic acid sepharose $6 \mathrm{~B}$ column. The column had been washed with 2 column volumes of distilled water and then equilibrated with 5 column volumes of Binding buffer. After loading of the supernatant, the column was washed with 10 column volumes of Binding buffer and then with the same volume of Binding buffer containing $150 \mathrm{mM}$ imidazole. The E3 mutant was eluted with Binding buffer containing $500 \mathrm{mM}$ imidazole. The purification steps were analyzed by the SDS-polyacrylamide gel electrophoresis as shown in Fig. 1 (Srisuparbh, et al., 2003; Dhuna et al., 2005).

E3 assay and spectroscopic study. E3 activity was assayed at $37^{\circ} \mathrm{C}$ in $50 \mathrm{mM}$ potassium phosphate buffer ( $\mathrm{pH} \mathrm{8.0)}$ containing 1.5 $\mathrm{mM}$ EDTA. The activity was recorded spectrophotometrically by observing the reduction of $\mathrm{NAD}^{+}$at $340 \mathrm{~nm}$ with a SPECORD200 spectrophotometer (Analytik Jena AG). One unit of activity is defined as $1 \mu \mathrm{mol}$ of $\mathrm{NAD}^{+}$reduced per min.

The UV-visible absorption spectra were recorded from $350 \mathrm{~nm}$ to $550 \mathrm{~nm}$ using the same spectrophotometer. The fluorescence spectra were recorded using a FP-6300 spectrofluorometer (Jasco Inc.). Samples were excited at $296 \mathrm{~nm}$ and the emissions were recorded from $305 \mathrm{~nm}$ to $580 \mathrm{~nm}$. The data were transferred to an ASCII file and the spectra were drawn using the MicroCal Origin program (Photon Technology International).

\section{Results and Discussion}

The comparison of amino acid sequences around the active disulfide center regions of human E3 and the corresponding residues of other E3s from various sources is shown in Table 1. The 10th Ile residue is highly conserved in most E3s (97\% conservation). Only E3 from Pseudomonas putida (lpd3) has a Met residue at the site instead of the Ile. It is also located near the active disulfide center Cys residues. The active disulfide center of E3 is critical in its catalytic function. The center bond should be broken during the catalysis to form an 
Table 1. Comparison of amino acid sequences around active disulfide center regions of dihydrolipoamide dehydrogenases (E3) from various sources. The Ile-51 and active disulfide Cys residues in human E3 and the corresponding residues in other E3s are highlighted by italic bold letters

\begin{tabular}{ll}
\hline \multicolumn{1}{c}{ Source } & Amino acid sequence \\
\hline Human & GGTCLNVGCIP \\
Trypanosoma brucei & GGTCLNVGCIP \\
Saccharomyces cerevisiae & GGTCLNVGCIP \\
Pseudomonas putida (lpdg) & GGTCLNVGCIP \\
Azotobacter vinelandii & GGTCLNVGCIP \\
Escherichia coli & GGTCLNVGCIP \\
Haemophilus influenzae & GGTCLNVGCIP \\
Sus scrofa & GGTCLNVGCIP \\
Canis familiaris & GGTCLNVGCIP \\
Mus musculus & GGTCLNVGCIP \\
Trypanosoma brucei & GGTCLNVGCIP \\
Trypanosoma cruzi & GGTCLNVGCIP \\
Pisum sativum & GGTCLNVGCIP \\
Manduca sexta & GGTCLNVGCIP \\
Schizosaccharomyces pombe & GGTCLNVGCIP \\
Pseudomonas putida (lpd3) & GGTCLNVGCMP \\
Pseudomonas fluorescens & GGTCLNVGCIP \\
Rhodobacter capsulatus & GGTCLNVGCIP \\
Alcaligenes eutrophus & GGTCLNVGCIP \\
Bacillus stearothermophilus & GGTCLNVGCIP \\
Bacillus subtilis & GGTCLNVGCIP \\
Staphylococcus aureus & GGTCLNVGCIP \\
Vibrio parahaemolyticus & GGVCLNVGCIP \\
Mycoplasma pneumoniae & GGVCLNVGCIP \\
Mycoplasma genitalium & GGVCLNVGCIP \\
Chlamydia trachomatis & GGTCLNRGCIP \\
Chlamydia pneumoniae & GGTCLNRGCIP \\
Halobacterium volcanii & GGTCLNHGCIP \\
Bacillus subtilis (acol) & GGICLNWGCIP \\
Bacillus subtilis (bfmbc) & GGTCLNEGCIP \\
Synechocytis sp. & GGTCLHKGCIP \\
Acholeplasma laidlawii & GGTCVNRGCIP \\
Zymomonas mobilis & GGVCVNWGCIP \\
\hline
\end{tabular}

intermediate with substrate. To examine the importance of this highly conserved Ile residue in E3 structure and function, the corresponding Ile-51 in human E3 was substituted with Ala by site-directed mutagenesis.

Site-directed mutagenesis and purification of the mutant were performed as described in Materials and Methods. Two PCR generated a whole E3 DNA fragment containing the mutation of Ile-51 (ATT) to Ala (GCT). The fragment was digested with Mly113I and EcoRI and ligated with a human E3 mutant expression vector, pPROEX-1:E3(S-79 $\rightarrow$ C), which had been digested with Mly113I and EcoRI to remove the corresponding normal Mly113I /EcoRI fragment. The

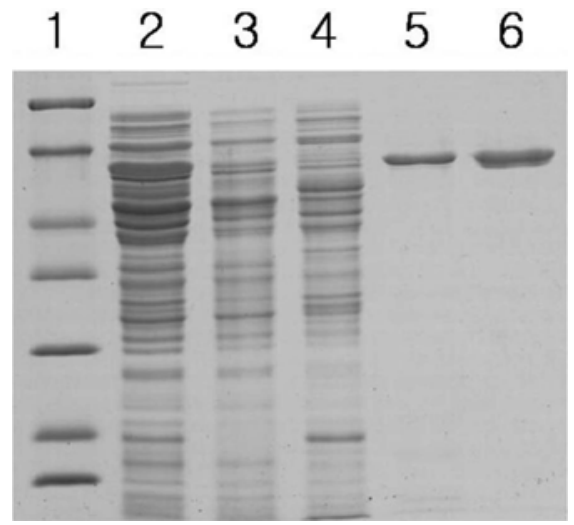

Fig. 1. SDS-polyacrylamide gel for the purification of the Ala-51 mutant human E3. Lane 1, molecular weight markers (from top to bottom, $\beta$-galactosidase $116.3 \mathrm{kDa}$, bovine serum albumin $66.2 \mathrm{kDa}$, ovalalbumin $45.0 \mathrm{kDa}$, lactate dehydrogenase $35.0 \mathrm{kDa}$, REase Bsp981 $25 \mathrm{kDa}, \quad \beta$-lactoglobulin $18.4 \mathrm{kDa}$, lysozyme $14.4 \mathrm{kDa}$ ); lane 2, supernatant; lane 3, flow-through; lane 4, Binding buffer containing $150 \mathrm{mM}$ imidazole; lane 5, binding buffer containing $500 \mathrm{mM}$ imidazole; lane 6 , previously purified recombinant human E3 as a control.

expression vector, pPROEX-1:E3(S-79 $\rightarrow$ C) was used since its $\mathrm{XbaI}$ site had been removed from the previous mutation (Kim, 1999a). The ligation resulted in the construction of the human E3 Ala-51 mutant expression vector, pPROEX-1:E3 $(\mathrm{I}-51 \rightarrow \mathrm{A})$. Screening of the mutant expression vector was performed by an $X b a \mathrm{I}$ digestion since only the Ala-51 mutant expression vector, pPROEX-1:E3 (I-51 $\rightarrow$ A) had an XbaI site. The mutation of ATT (Ile-51) to GCT (Ala) in the human E3 coding region of the mutant expression vector, pPROEX1:E3 (I-51 $\rightarrow$ A) was confirmed by DNA sequencing. No other mutation was observed in the human E3 coding region of the mutant expression vector, pPROEX-1:E3 (I-51 $\rightarrow$ A).

The expression and purification of the mutant were performed as described in Materials and Methods. The expression of the mutant in $E$. coli was induced by adding IPTG. After equilibration with Binding buffer, supernatant solution was applied to the column. E. coli proteins were washed out with Binding buffer and then with Binding buffer containing $150 \mathrm{mM}$ imidazole. The mutant was eluted with Binding buffer containing $500 \mathrm{mM}$ imidazole. Since human E3 was a flavoprotein, its yellow color was a good indicator for the presence of human E3 protein during the purification. Purification steps were followed by SDS-polyacrylamide gel electrophoresis as shown in Fig. 1. The gel showed that the mutant was highly purified.

The E3 activity of the mutant was determined as $6.1 \mathrm{unit} / \mathrm{mg}$ at saturated substrate concentrations of $2 \mathrm{mM}$ dihydrolipoamide and $3 \mathrm{mM} \mathrm{NAD}{ }^{+}$at $37^{\circ} \mathrm{C}$. This value was about 100 -fold lower than that of normal human E3 activity determined at the same condition. Even though it was difficult to obtain the detailed kinetic parameters due to the low activity and instability of the mutant, this 100-fold decreased E3 activity of 


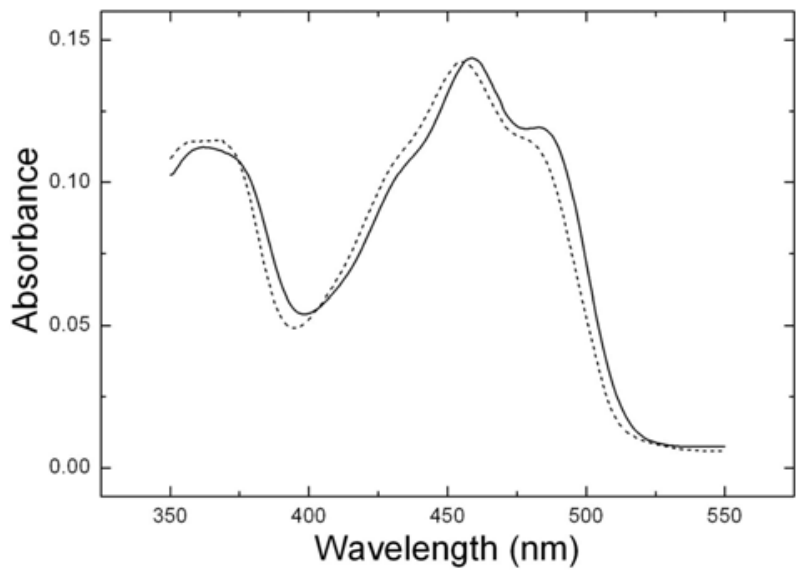

Fig. 2. UV-visible spectra of the Ala-51 mutant $(13.0 \mu \mathrm{M}$, solid line) and normal $(12.9 \mu \mathrm{M}$, dot line) recombinant human E3s. The spectra were recorded using Shimazu UV 160A spectrophotometer and the data from $350 \mathrm{~nm}$ to $550 \mathrm{~nm}$ were transferred to an ASCII file and the spectra were drawn using the MicroCal Origin program.

the Ala-51 mutant clearly indicated that the conservation of the Ile-51 residue in human E3 was very important to the catalytic function of the enzyme.

The active disulfide center should be broken and reformed during the E3 catalysis. It has been known that the disulfide bond structure of the active disulfide center is unusual (Mattevi et al., 1991). The $\mathrm{C}^{\alpha}-\mathrm{C}^{\alpha}$ distance between the two Cys residues of the active disulfide center is relatively short and the torsion angle values along the Cys side chains are unique. This unusual structure gives some geometric strain which may be necessary for the proper catalytic function of the active disulfide center during the E3 catalysis. The active disulfide center of human E3 is formed between the Cys-45 and Cys-50. The Ile-51 is the next residue of the Cys-50. The mutation of Ile-51 to Ala results in the removal of three methyl groups at the side chain of the residue- 51 of human E3. This absence of three methyl groups at the residue-51 could influence the proper conformation of the active disulfide center region of human E3 so that the proper function of the active disulfide center in the efficient catalytic processes of human E3 could be interfered. This interference could be the reason for the observed large decrease in the E3 activity of the mutant.

To examine any conformational changes occurring in the mutant due to the mutation, UV-visible absorption and fluorescence spectra were observed as described in Materials and Methods. Human E3 contains one FAD as a prosthetic group in each subunit. It therefore shows a characteristic UVvisible absorption spectrum of flavoproteins as shown in Fig. 2 (dot line). It has two peaks, one broad peak from $358 \mathrm{~nm}$ to $370 \mathrm{~nm}$ and the other at $455 \mathrm{~nm}$. The overall shape of the mutant spectrum (solid line) was very similar to that of the normal human E3 spectrum (dot line). However, the overall

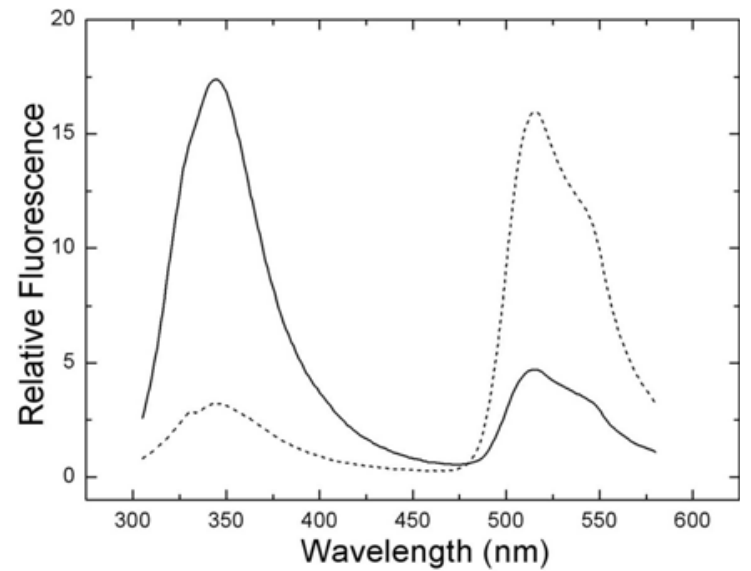

Fig. 3. Fluorescence spectra of the Ala-51 mutant $(16.5 \mu \mathrm{M}$, solid line) and normal $(10.5 \mu \mathrm{M}$, dot line) recombinant human E3s. Enzymes were excited at $296 \mathrm{~nm}$ and the emissions were observed from $305 \mathrm{~nm}$ to $580 \mathrm{~nm}$. The data were transferred to an ASCII file and the spectra were drawn using the MicroCal Origin program.

spectrum of the mutant was slightly shifted to the right about $3 \mathrm{~nm}$ as shown in Fig. 2, indicating that conformational changes could occur in the mutant.

Further evidence for the conformational changes in the mutant came from the fluorescence study, which was very sensitive to conformational changes in proteins. As shown in Fig. 3, two fluorescence emissions were observed for human E3. The first emission from $305 \mathrm{~nm}$ to $400 \mathrm{~nm}$ is mainly due to tryptophans. The second emission from $480 \mathrm{~nm}$ to over 550 $\mathrm{nm}$ is due to FAD. When the fluorescence spectra of the mutant and wild-type E3s were compared, a noticeable difference was found in the ratio between relative intensities of the first and second fluorescence emissions. The ratio (about 0.3) between relative intensities of the first and second fluorescence emissions of the mutant (solid line) was much lower than that (about 5) of the wild-type enzyme (dot line). This observed difference in the ratio between the relative intensities of the first and second fluorescence emissions of E3s was mainly due to a large increase in the intensity of the first emission of the mutant. This difference in the fluorescence spectrum patterns between the mutant and wildtype enzymes again implied that slight conformational changes could occur in the mutant. A similar high increase in the first fluorescence emission had been reported in the spectroscopic studies of other human E3 mutants. One was Glu-457 to Gln mutant (Liu et al., 1995) and the other was a mutant lacking the last five amino acids (Kim, 1999b). The conformational changes, which led to the previously altered UV-visible spectrum of the mutant, could cause these alterations in fluorescence spectrum of the mutant.

From these results, the following suggestions can be made. First, the conservation of the Ile-51 residue in human E3 is very important to the efficient catalytic function of the 
enzyme. Second, the substitution of Ile-51 with Ala can cause the conformational changes in human E3. Third, these conformational changes can reduce largely the E3 activity of human E3.

Acknowledgments The author thanks Dr. Mulchand S. Patel (State University of New York, Buffalo) for a generous gift of an E. coli XL1-Blue containing a human E3 expression vector. The author is grateful to Dr. Tai Jong Kang (Daegu University) for providing a fluorometer. The author thanks Jeong-Wha Han for technical helps. This work was supported in part by the Daegu University Research Grant, 2004.

\section{References}

Dhuna, V., Bains, J. S., Kamboj, S. S., Sinngh, J., Shanmugavel and Saxena, A. K. (2005) Purification and characterization of a lectin from Arisaema tortuosum Schott having in-vitro anticancer activity against human cancer cell lines. J. Biochem. Mol. Biol. 38, 526-532.

Jentoft, J. E., Shoham, M., Hurst, D. and Patel, M. S. (1992) A structural model for human dihydrolipoamide dehydrogenase. Proteins: Struct. Funct. Genet. 14, 88-101.

Kim, H. (1999a) Protein engineering of an artificial intersubunit disulfide bond linkage in human dihydrolipoamide dehydrogenase. J. Biochem. Mol. Biol. 32, 76-81.

Kim, H. (1999b) Deletion of the last five amino acid residues in human dihydrolipoamide dehydrogenase. Bull. Korean Chem. Soc. 20, 1221-1224.

Kim, H. (2001) Consensus sequence for the active site disulfide bond region of dihydrolipoamide dehydrogenase with known primary structure. J. Natl. Sci. 18, 23-29.

Kim, H. (2002) Activity of human dihydrolipoamide dehydrogenase is reduced by mutation at threonine-44 of FAD-binding region to valine. J. Biochem. Mol. Biol. 35, 437-441.

Kim, H. (2005) Asparagine-473 residue is important to the efficient function of human dihydrolipoamide dehydrogenase. $J$. Biochem. Mol. Biol. 38, 248-252.

Kim, H., Liu, T.-C. and Patel, M. S. (1991) Expression of cDNA sequences encoding mature and precursor forms of human dihydrolipoamide dehydrogenase in Escherichia coli. J. Biol. Chem. 266, 9367-9373.

Kim, H. and Patel, M. S. (1992) Characterization of two sitespecifically mutated human dihydrolipoamide dehydrogenases (His-452 $\rightarrow$ Gln and Glu-457 $\rightarrow$ Gln). J. Biol. Chem. 267, 5128-5132.

Liu, T., Korotchkina, L. G., Hyatt, S. L., Vettakkorumakankav, N. N. and Patel, M. S. (1995) Spectroscopic studies of the characterization of recombinant human dihydrolipoamide dehydrogenase and its site-directed mutants. J. Biol. Chem. 270, 15545-15550.

Mattevi, A., Obmolova, G., Sokatch, J.R., Betzel, C., Hol, W. G. J. (1992) The refined crystal structure of Pseudomonas putide lipoamide dehydrogenase complexed with $\mathrm{NAD}^{+}$at $2.45 \AA$ resolution. Proteins Struct. Funct. Genet. 13, 336-351.

Pons, G., Raefsky-Estrin, C., Catothers, D. J., Pepin, R. A., Javed, A. A., Jesse, B. W., Ganapathi, M. K., Samols, D. and Patel, M. S. (1988) Cloning and cDNA sequence of the dihydrolipoamide dehydrogenase component of human $\alpha$ ketoacid dehydrogenase complexes. Proc. Natl. Acad. Sci. USA 85, 1422-1426.

Reed, L. J. (1974) Multienzyme complexes. Acc. Chem. Res. 7, 40-46.

Schierbeek, A. J., Swarte, M. B. A., Dijkstra, B. W., Vriend, G., Reed, R. J., Hol, W. G. J. and Drenth, J. (1989) X-ray structure of lipoamide dehydrogenase from Azotobacter vinelandii determined by a combination of molecular and isomorphous replacement techniques. J. Mol. Biol. 206, 365-379.

Srisuparbh, D., Klinbunga S., Wongsiri, A. and Sittipraneed, S. (2003) Isolation and characterization of major royal jelly cDNAs and proteins of the honey bee (Apis cerana). $J$. Biochem. Mol. Biol. 36, 572-579.

Thieme, R., Pai, E. F., Schirmer, R. H. and Schulz, G. E. (1981) Three-dimensional structure of glutathione reductase at $2 \AA$ resolution. J. Mol. Biol. 152, 763-782.

Walker, J. L. and Oliver, D. J. (1986) Glycine decarboxylase multienzyme complex. Purification and partial characterization from leaf mitochondria. J. Biol. Chem. 261, 2214-2221.

Williams, C. H. Jr. (1976) Flavin containing dehydrogenases; in Enzymes, Boyer, P. (ed.), pp. 89-173, Academic Press, New York, USA. 